\title{
Using domperidone to increase breast milk supply: a clinical practice survey of Australian neonatal units
}

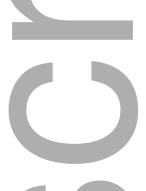

Original article

Authors and Affiliations:

1. Christine E Gilmartin BPharm(Hons), GradCertPharmPrac

The Royal Women's Hospital Pharmacy Department, The Royal Women's Hospital; Parkville, Victoria, Australia

2. Lisa H Amir, PhD, MBBS, MMed

Judith Lumley Centre, La Trobe University, Melbourne, Victoria, Australia Breastfeeding Service, Royal Women's Hospital, Melbourne, Victoria, Australia

3. Marene Ter, MClinPharm, BPharm(Hons)*

The Royal Women's Hospital Pharmacy Department, The Royal Women's Hospital; Parkville, Victoria, Australia

Pharmacy Department, The Northern Hospital, Epping, Victoria, Australia

4. Luke E Grzeskowiak, PhD, BPharm(Hons)

Adelaide Medical School, Robinson Research Institute, The University of Adelaide, Adelaide, South Australia, Australia \& SA Pharmacy, Flinders Medical Centre, SA Health, Adelaide, South Australia, Australia

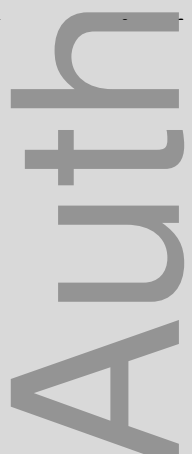

Corresponding Authors:

Christine Gilmartin

c/o Pharmacy Department, Level 1, The Royal Women's Hospital

Cnr. Grattan St. \& Flemington Rd

Parkville, 3052

Victoria, Australia

T: +61 383453187

E-mail: Christine.Gilmartin@thewomens.org.au 


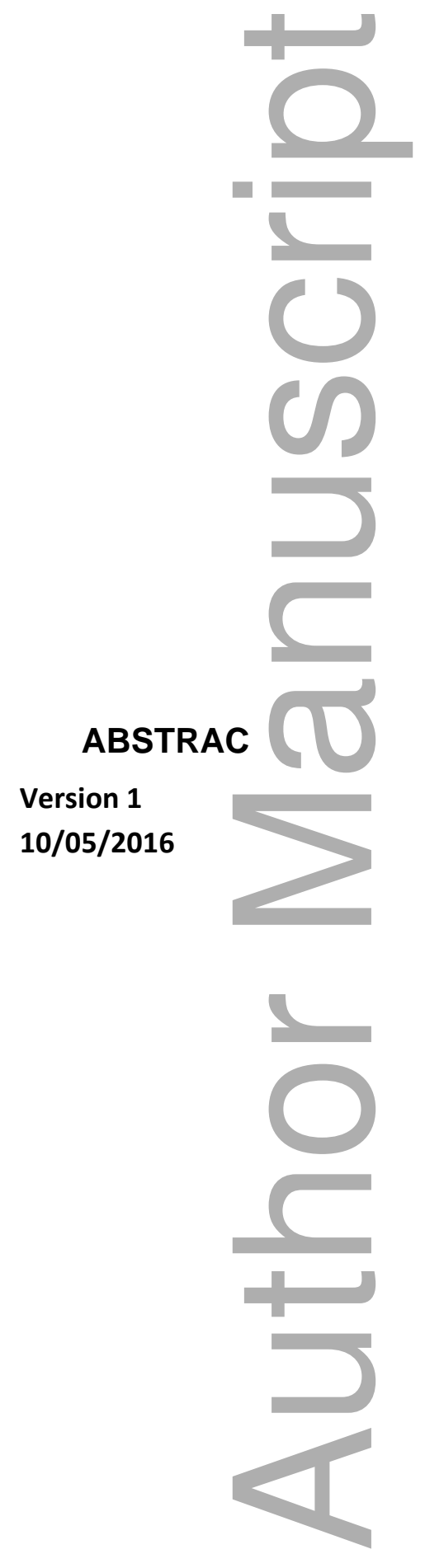

This article is protected by copyright. All rights reserved 
Received Date : 12-May-2016

Revised Date : 22-Aug-2016

Accepted Date : 05-Sep-2016

Article type : Research Article

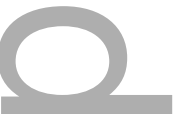

Abstract

Background: Domperidone may be used off-label to increase breast-milk supply. In the absence of manufacturer dosage recommendations, there is a need to compare the clinical practice guidelines used in Australian hospitals.

Aim: To compare existing clinical practice guidelines regarding the use of domperidone for increasing maternal breast milk supply within Australian neonatal units.

Methods: An electronic survey was distributed between April to July 2015 to relevant staff at each Level III neonatal unit (NNU) across Australia.

Results: Responses were received from 16 of 22 (73\%) NNUs. All 16 (100\%) NNUs considered domperidone their first line pharmacological agent of choice in the management of low milk supply, with 12 (75\%) having a clinical guideline. Of the NNUs with a guideline, 11 (92\%) recommended a standard maintenance dose of $10 \mathrm{mg}$ three times daily (TDS). Duration of treatment was specified in $10(83 \%)$ guidelines. 7 of $10(70 \%)$ recommend a minimum duration of at least two weeks treatment before evaluating a response. 3 of $11(27 \%)$ guidelines that recommended 10mg TDS permit an increase to 20mg TDS depending on response. 10 of 12 (83\%) guidelines have been modified in the previous 2-3 years, with eight revising their maximum dose to $30 \mathrm{mg} / \mathrm{day}$, one limiting treatment duration to seven days, and one removing the authority of neonatal unit doctors to prescribe domperidone.

Conclusion: While all Australian neonatal units consider domperidone first-line for pharmacological management of low milk supply, variability across clinical guidelines may suggest a lack of reliable evidence or awareness of evidence on which to base practice.

\section{Introduction}

This article is protected by copyright. All rights reserved 
The maternal and infant benefits of breastfeeding are well recognised, with breast milk considered the optimal form of nutrition to support the growth and development of term and preterm infants. ${ }^{1}$ Preterm infants are especially vulnerable to infections, including the potentially fatal necrotizing enterocolitis, if exposed to non-human milks. ${ }^{2}$ Mothers who give birth prematurely often struggle especially to establish an adequate milk supply. Their breasts do not have the complete development experienced by mothers giving birth at term and their infants may be unable to feed directly at the breast. ${ }^{3}$

While non-pharmacological strategies for improving milk supply are considered first-line, in situations where these strategies are not effective, pharmacological intervention using medications known as galactagogues may be recommended to stimulate breast milk production. ${ }^{4,5,6}$ One of the best-studied galactagogues is domperidone, a dopamine receptor antagonist which is thought to increase breast milk supply by increasing serum prolactin levels. Data based on a small number of randomized controlled trials indicate that domperidone is associated with modest improvements in breast milk supply, and also that there are few maternal adverse effects and no reported neonatal adverse effects. ${ }^{7-8}$

Despite data to support its use, lactation support is not a marketed indication for domperidone. ${ }^{9}$ This means that domperidone is used "off-label", with an absence of any manufacturer recommendations regarding its safe and appropriate use in this setting. ${ }^{5,9}$ The European Medicines Agency's (EMA) Pharmacovigilance Risk Assessment Committee (PRAC) recently published recommendations restricting domperidone use to a maximum dose of 30mg daily as well as warning against use for longer than one week. ${ }^{10}$ There are no comprehensive published guidelines used universally that elaborate further. As a result, domperidone dosage recommendations may be instead based on a combination of prescriber preference, anecdotal experience, external guidelines from breastfeeding organisations, or existing published studies. ${ }^{6}$ Domperidone dosing regimens that appear to be used in small randomised clinical trials often range from $10 \mathrm{mg}$ to $20 \mathrm{mg}$ three times daily (TDS), with durations varying from 1-4 weeks. ${ }^{5,11}$ Given the varied origins of information which recommendations may be based on, there is a need to compare the resulting clinical practice guidelines regarding the use of domperidone for increasing breast milk supply across Australian hospital neonatal units. Therefore, the aim of this study was to compare existing clinical practice guidelines regarding the use of domperidone for increasing breast milk supply within Australian neonatal units.

\section{Method}

An email survey regarding the use of domperidone for increasing breast milk supply was distributed between April to July 2015 to lactation consultants (health professionals who hold an 
International Board Certified Lactation Consultant qualification (IBCLC) and specialise in training and assisting mothers to breastfeed) and pharmacists at each Level III neonatal unit (NNU) across Australia (22 units). ${ }^{12}$ Australian Level III NNUs were identified from the Australian and New Zealand Neonatal Network (ANZNN), and defined as tertiary centres of intensive care for newborns requiring continuous life support and complex multidisciplinary services. ${ }^{13}$ Pharmacists and lactation consultants were selected as the appropriate respondents for this survey, as due to the nature of their role they would be aware of the presence of a clinical guideline regarding the use of domperidone, and the broader prescribing habits for domperidone, within their workplace. Surveyed sites included both metropolitan and regional centres, and all were both public and teaching hospitals. Four main questions were included in the survey which focused on: first line pharmacological management of low milk supply in the NNU, availability of clinical guidelines, recent changes to clinical guidelines, and the evaluation and documentation of patient suitability for pharmacological management when prescribing. One survey was completed for each neonatal unit by a lactation consultant or a pharmacist, with a copy of current and previous clinical guidelines (where applicable) requested for examination. Survey responses were entered into a Microsoft Excel® spreadsheet and analyzed using descriptive statistics to identify differences in practice. A complete copy of the survey is available on request from the corresponding author.

While a full ethics approval was not required by the design of this study, The Royal Women's Hospital HREC was notified of the project and intent to contact staff within each NNU. ${ }^{14}$

\section{Results}

Responses were received from 16 of 22 (73\%) Australian Level III NNUs, which accounted for 716 of $911(79 \%)$ nursery beds in 2015. Of the respondents, 11 were pharmacists and 5 were lactation consultants. All $16(100 \%)$ Australian NNUs considered domperidone the first line pharmacological agent of choice in the management of low milk supply, with 12 (75\%) NNUs having a specific clinical guideline incorporating its use. Of the NNUs with a clinical guideline, $11(92 \%)$ recommended a standard maintenance dose of 10mg TDS, with one guideline (8\%) recommending a standard maintenance dose of 20mg TDS. Duration of treatment was specified in $10(83 \%)$ guidelines, all with a recommended dosing regimen of $10 \mathrm{mg}$ TDS, of which the majority of guidelines ( 7 of 10; 70\%) recommended a minimum duration of at least two weeks treatment before evaluating a response (Figure 1). Furthermore, the majority of clinical guidelines (10 of $12 ; 83 \%$ ) recommend tapering the maintenance dose before ceasing. The tapering dose regimens differed slightly across guidelines, with tapering to occur over 7 to 14 days. In all cases, the tapering dose involved decreased from a dosing frequency of three times 
daily to twice daily, and then once daily before ceasing. Of the 11 clinical guidelines that recommended a maintenance dose of $10 \mathrm{mg}$ TDS, 3 (27\%) mentioned the possibility of increasing to $20 \mathrm{mg}$ TDS depending on response. In one NNU guideline a maternal electrocardiogram (ECG) was recommended prior to increasing the dose to 20mg TDS.

One of the most notable findings was that changes within the previous 2-3 years have been made to 10 of $12(83 \%)$ clinical guidelines, with 8 revising their maximum dose to $30 \mathrm{mg} /$ day, one limiting the duration of treatment to seven days, and one removing the ability of neonatal unit doctors to prescribe domperidone to mothers of infants in their care. Approximately a third ( 5 of $16 ; 31 \%$ ) of NNUs formally documented the evaluation of patient risk factors prior to beginning domperidone therapy, with 2 of 16 (12.5\%) NNUs utilizing a specific domperidone prescribing checklist for doctors to more safely facilitate supply. These checklists included questions pertaining to the appropriate trial of non-pharmacological strategies and presence of contraindications or precautions such as maternal allergies, medical conditions (e.g. renal/hepatic impairment, cardiac disease, hyperprolactinoma), and medication use (e.g. medications that prolong the QT interval or inhibit CYP3A4). The checklists also required documentation that the use, risks and benefits of domperidone have been described to the mother by the prescriber.

\section{Discussion}

Variability exists across Australian neonatal units with respect to the use of domperidone for increasing breast milk supply. Such variations in clinical practice guidelines also highlight the lack of adequate evidence to support many clinical practice recommendations.

Data based on a small number of randomised controlled trials indicate that domperidone is associated with modest improvements in breast milk supply. ${ }^{7-8}$ Domperidone use in studies such as Knoppert et al and Wan et al have established efficacy in increasing breast-milk supply using both 30mg and 60mg daily. ${ }^{5,11}$ However, participant numbers have been small, domperidone was taken for differing time periods ranging from 1-4 weeks, and a statistically significant doseresponse relationship has not yet been demonstrated. ${ }^{5,11}$ This data supports the use of domperidone, as identified in this survey, as a first line pharmacological agent for managing low milk supply. However, the manner in which domperidone is used in clinical practice has remained unknown. ${ }^{15}$ Despite being developed from the same published evidence, this clinical practice survey has identified substantial variations across Australian NNUs. Such variations not only indicate an urgent need for further research in areas of uncertainty, but also highlight 
the requirement for the improved implementation of existing evidence into clinical practice where sound evidence is available.

Initiating Treatment

Based on currently available evidence, the optimal timing in which to initiate domperidone remains largely unconfirmed. ${ }^{15}$ While none of the guidelines in this survey specifically identified an appropriate timing postpartum at which domperidone is recommended to be commenced, there was a positive emphasis on the requirement for a thorough trial of nonpharmacological strategies first.

\section{Maternal Risk Assessment}

Only a few NNUs, once the decision to initiate treatment with domperidone was made, reported having a formal process for documenting the evaluation of maternal risk factors. One of the major concerns regarding the use of domperidone relates to its potential to prolong the QT interval, which places susceptible users at a significantly increased risk of fatal cardiac arrhythmias. ${ }^{16}$ These findings have led regulatory bodies around the world to issue warnings regarding the use of domperidone. These warnings have generated significant controversy and continued debate regarding the relative risks and benefits of domperidone use for the management of low milk supply. ${ }^{17,18,19}$ Notably, even though any increased risk of sudden cardiac death is likely to be very small and extremely rare, it is not possible to rule out any increased risk among breastfeeding women taking domperidone. One American hospital has implemented mandatory cardiac examinations by auscultation prior to initiation of domperidone treatment. ${ }^{20}$ While we do not assume that our study finding implies that the remaining NNUs did not appropriately assess mothers prior to prescribing, we do agree with the practice of formal documentation to facilitate prescriber compliance. This should be considered best practice, considering its off-label medication status, and implemented by way of a standardized prescribing checklist. Although one hospital recommended baseline ECG monitoring prior to increasing the domperidone dose to $20 \mathrm{mg}$ three times daily, there is insufficient evidence with which to justify this additional screening expense.

\section{Changes in Practice}

Despite few NNUs reporting having a formal process for evaluating and documenting maternal risk factors, the majority of NNUs have recently altered their domperidone guideline in the 
previous 2-3 years. In all of these cases, the guidelines were modified to restrict the use of domperidone, either by reducing the recommended maintenance dose from $60 \mathrm{mg} / \mathrm{day}$ to $30 \mathrm{mg} / \mathrm{day}$, or by reducing the duration of treatment to seven days. While such changes may appear consistent with the regulatory warnings previously discussed, they could be considered in contrast to the published literature describing domperidone use for lactation ${ }^{21,7-8}$.

\section{Maintenance Dose and Duration of Treatment}

While most guidelines recommend a maintenance dose of $10 \mathrm{mg}$ three times daily, variability was observed with respect to the duration of treatment, ranging from five days to six weeks or longer. In some cases, the treatment duration was purely based on individual response, with no specific timelines set. According to previous RCTs, while a treatment effect is likely to be observed within seven days of treatment, maximal treatment effect is unlikely to be achieved until 2 to 4 weeks $^{8}$. While it is preferred that treatment regimens be tailored to the individual's needs, guidelines that recommend $\leq 7$ day courses of domperidone are likely to be too short to elicit desired benefits. Such short courses are particularly unsuitable in mothers of extremely preterm infants where supply difficulties are expected to be ongoing.

\section{Higher Doses}

Although only one guideline recommended a standard maintenance dose of 20mg TDS, 3 of 12 (33\%) guidelines mentioned the possibility of increasing to 20mg TDS depending on individual response. Previous studies point towards the potential for a dose response relationship and the possibility of greater response with higher doses ${ }^{5,11}$, but these differences have not yet been demonstrated to be significantly statistically different. In light of this, while a routine maintenance dose of $20 \mathrm{mg}$ three times daily cannot be advocated as first line management, evidence supports the use of an escalated dose where after careful expert review it may be considered necessary.

\section{Dose Tapering}

Differences in dose tapering recommendations were also identified across the guidelines. Despite not being well studied, the practice of dose tapering is commonly recommended based on anecdotal reports of a sudden drop in milk supply following abrupt cessation of domperidone. ${ }^{15}$ Furthermore, while the risks of medication withdrawal are likely to be low following common treatment regimens, domperidone withdrawal has been reported following 
abrupt cessation in a women taking domperidone $20 \mathrm{mg}$ four times daily for eight months. ${ }^{22}$ As a result, the recommendation for dose tapering appears reasonable with the caveat that no data is available to guide the optimal approach.

\section{Strengths and limitations}

This survey received a relatively high response rate from Australian units, with responding NNUs accounting for a large proportion of level III nursery beds across Australia. However, a major limitation of this study is that it reflects data obtained only from clinical practice guidelines. The nature of guidelines is such that they guide, but do not necessarily dictate, practice. $^{23}$ It is entirely possible that some women are provided treatment which does not comply with local clinical guideline recommendations. Similarly, among NNUs at which domperidone clinical guidelines were not available, it is not possible to accurately elucidate from such a survey as to what constitutes common practice within those NNUs. Therefore, the results of this survey potentially may not capture the full extent of the variability with which domperidone is currently used in clinical practice. A further limitation is that this survey was not able to determine the frequency with which domperidone is used within the various NNUs. A previous audit of clinical practice at an Australian tertiary level teaching hospital identified that domperidone was dispensed to at least $3.6 \%$ of mothers who delivered their infants at that hospital. ${ }^{6}$ In around $50 \%$ of cases the infants of mothers dispensed domperidone were admitted

to the NNU. ${ }^{6}$ A subsequent analysis of data from the same cohort identified that the percentage of mothers dispensed domperidone was well over $10 \%$ among those with a gestational age at delivery of less than 35 weeks, rising to as high as $50 \%$ or more at less than 28 weeks gestation. ${ }^{24}$ Identifying how such rates of domperidone use differ across the various NNUs, as well as their subsequent infant feeding outcomes, may be of particular interest for further studies.

In conclusion, it was found that all surveyed Australian NNUs were consistent in considering domperidone their first-line agent for increasing breast-milk supply, and 92\% recommended a standard maintenance dose of 10mg TDS. However, some variability regarding recommendations for domperidone in increasing breast milk supply persists between them, which may suggest a lack of reliable evidence or awareness of evidence to guide clinical practice. Rationalisation of in-house clinical practice guidelines to optimize domperidone use would be prudent in order to improve outcomes for mothers and infants in addition to minimising safety risks. Further research is required to address key evidence gaps in the dose, 
treatment duration, use of dose tapering, methods to identify women most likely to receive benefit from domperidone, identifying the most effective treatment initiation regimen, and investigating the long-term breastfeeding outcomes in mothers taking domperidone.

\section{References:}

1. Stuebe AM, Schwarz EB. The risks and benefits of infant feeding practices for women and their children. J Perinatol 2010; 30: 155-162.

2. Maayan-Metzger A, Avivi S, Schushan-Eisen I, Kuint J. Human milk versus formula feeding among preterm infants: short-term outcomes. Am J Perinatol. 2012; 29: 121-6.

3. Amir LH. Breastfeeding: managing 'supply' difficulties. Aust Fam Physician. 2006; 35: 686-689.

4. Zuppa, AA, Sindico P, Orchi C, Carducci C, Cardiello V, Romagnoli C. Safety and efficacy of galactogogues: substances that induce, maintain and increase breast milk production. J Pharm Pharm Sci. 2010; 13(2): 162-74.

5. Wan, E W-X, Davey K, Page-Sharp M, Hartmann PE, Simmer K, Ilett KF. Dose-effect of domperidone as a galactogogue in preterm mothers with insufficient mild supply, and it's transfer into milk. Br J Clin Pharmacol. 2008; 66(2): 283-289.

6. Grzeskowiak LE, Lim SW, Thomas AE, Ritchie U, Gordon AL. Audit of domperidone use as a galactogogue at an Australian tertiary teaching hospital. J Hum Lact; 2013; 29(1):32-7.

7. Osadchy A, Moretti ME, Koren G. Effect of domperidone on insufficient lactation in puerperal women: a systematic review and meta-analysis of randomized controlled trials. Obstet Gynecol Int; 2012; 1-7.

8. Donovan TJ, Buchanan K. Medications for increasing milk supply in mothers expressing breastmilk for their preterm hospitalised infants. Cochrane Database Syst Rev; 2012; 3, CD005544.

9. Motilium Product Information - Janssen-Cilag; 2015; MIMS Online; MIMS Australia.

10. European Medicines Agency. PRAC recommends restricting use of domperidone. London, UK. Available from:

[http://www.ema.europa.eu/docs/en_GB/document_library/Referrals_document/Domper idone_31/Recommendation_provided_by_Pharmacovigilance_Risk_Assessment_Comm ittee/WC500162559.pdf]; Accessed: Apr 2014. 
11. Knoppert DC, Page A, Warren J, et al. The effect of two different domperidone doses on maternal milk production. J Hum Lact. 2013; 29(1)38-44.

12. Australian Breastfeeding Association. Lactation Consultants. Malvern East, VIC, Australia. Available from < www.breastfeeding.asn.au/roles/lactation-consultants $>$.

13. Intensive Care Unit. Australian Institute of Health and Welfare. Australian Government. Australia. Available from <http://meteor.aihw.gov.au/content/index.phtml/itemId/327234> .

14. Hui A CB. (Human Research and Ethics Committee, The Royal Womens Hospital, Parkville, Australia). Letter to: Marene Ter (The Royal Women's Hospital, Parkville Australia). 2015 Apr 20. 1 leaf. Located at: Pharmacy Department, Level 1, The Royal Women's Hospital, Cnr Flemington Rd \& Grattan St, Parkville, 3052, Australia.

15. Powers NG, Montgomery A, Academy of Breastfeeding Medicine Protocol Committee. ABM Clinical Protocol \#9: Use of galactogogues in initiating or augmenting the rate of maternal milk secretion (first revision January 2011). Breastfeed Med 2011; 6(1): 41-9.

16. Doggrell SA, Hancox JC. Cardiac safety concerns for domperidone, an antiemetic and prokinetic, and galactogogue medicine. Expert Opin Drug Saf; 2014; 13(1): 131138.Paul C, Zénut M, Dorut A, Coudore, MA; Vein, J; Cardot, JM; Balayssac,D. Use of domperidone as a galactagogue drug a systematic review of the benefit-risk ratio. J Hum Lact. 2015. 31(1): 57-63.

17. Grzeskowiak LE. Use of domperidone to increase breast milk supply: are women really dying to breastfeed? J Hum Lact. 2014. 30(4): 498-499.

18. Grzeskowiak LE, Amir LH. Use of domperidone to increase breast milk supply: further consideration of the benefit-risk ratio is required. J Hum Lact. 2015; 31(2): 312-316.

19. Bozzo P, Koren G, Ito S. Health Canada advisory on domperidone: Should I avoid prescribing domperidone to women to increase milk production? Can Fam Physician. 2012; 58: 952-953.

20. Haase B, Taylor SN, Mauldin J, Johnson TS, Wagner CL. Domperidone for Treatment of Low Milk Supply in Breast Pump-Dependent Mothers of Hospitalized Premature Infants: A Clinical Protocol. J Hum Lact. 2016; 32(2): 373-381.

21. Grzeskowiak, LE; Amir LH. Pharmacological management of low mild supply with domperidone: separating fact from fiction. MJA; 2014, 201(5): 257-258.

22. Papastergiou, J; Abdallah, M; Tran, A, Folkins, C. Domperidone withdrawal in a breastfeeding woman. Can Pharm J; 2013; 146(4):210-212.

23. Keiffer MR. Utilization of clinical practice guidelines: barriers and facilitators. Nur Clin 
North Am 2015; 50: 327-45.

24. Grzeskowiak, LE; Dalton, J; Fielder, A. Factors associated with domperidone use as a galactogogue at an Australian tertiary teaching hospital. J Hum Lact. 2015; 31: 249-253.

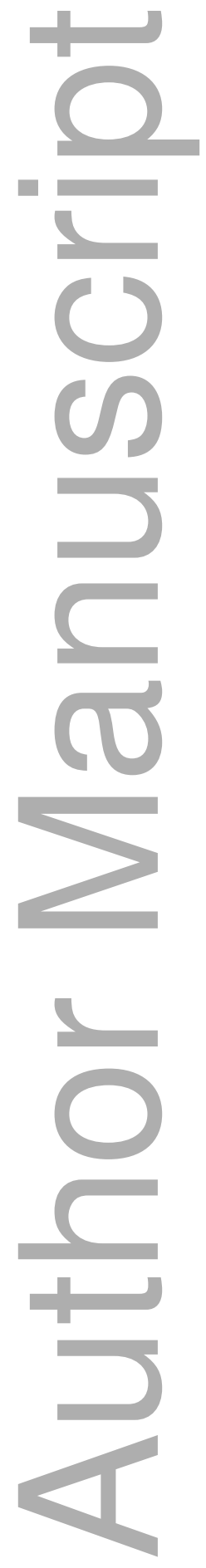




\section{Figure 1. Recommended Duration of Treatment with Domperidone According to Each Hospital Guideline}

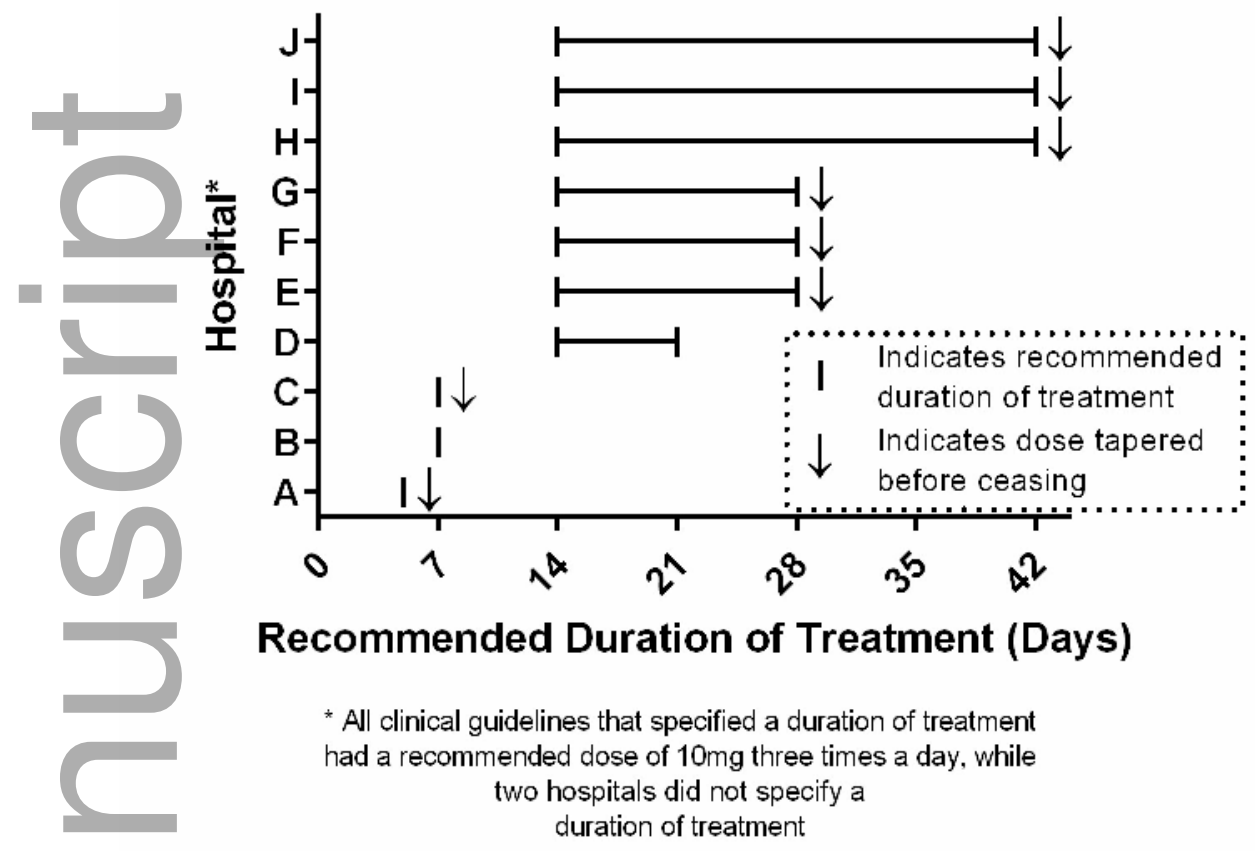

jppr_1289_f1.jpg 


\section{University Library}

\section{- M M I N E R VA A gateway to Melbourne's research publications}

Minerva Access is the Institutional Repository of The University of Melbourne

Author/s:

Gilmartin, CE;Amir, LH;Ter, M;Grzeskowiak, LE

Title:

Using domperidone to increase breast milk supply: A clinical practice survey of australian neonatal units

Date:

2017-12-01

Citation:

Gilmartin, C. E., Amir, L. H., Ter, M. \& Grzeskowiak, L. E. (2017). Using domperidone to increase breast milk supply: A clinical practice survey of australian neonatal units. Journal of Pharmacy Practice and Research, 47 (6), pp.426-430. https://doi.org/10.1002/jppr. 1289.

Persistent Link:

http://hdl.handle.net/11343/292488 